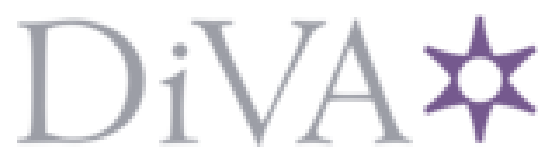

http://www.diva-portal.org

\title{
Preprint
}

This is the submitted version of a paper published in Development, Genes and Evolution.

Citation for the original published paper (version of record):

Oliveira, M., Liedholm, S., Lopez, J., Lochte, A., Pazio, M. et al. (2014)

Expression of arthropod distal limb-patterning genes in the onychophoran Euperipatoides kanangrensis.

Development, Genes and Evolution, 224(2): 87-96

http://dx.doi.org/10.1007/s00427-014-0466-z

Access to the published version may require subscription.

N.B. When citing this work, cite the original published paper.

Permanent link to this version:

http://urn.kb.se/resolve?urn=urn:nbn:se:uu:diva-223536 


\section{Expression of arthropod distal limb-patterning genes in the onychophoran Euperipatoides kanangrensis}

Marta Bastos Oliveira ${ }^{\dagger}$, Simon Eckerström Liedholm ${ }^{\dagger}$, Jordi Estefa Lopez ${ }^{\dagger}$, Annalena A Lochte ${ }^{\dagger}$, Magdalena Pazio ${ }^{\dagger}$, Jesus Pena Martin ${ }^{\dagger}$, Patrik Rödin Mörch ${ }^{\dagger}$, Seela Salakka $^{\dagger}$, Julia York ${ }^{\dagger}$, Andrew Yoshimoto ${ }^{\dagger}$, Ralf Janssen ${ }^{1 *}$

${ }^{1}$ Uppsala University, Department of Earth Sciences, Palaeobiology, Villavägen 16, 75236 Uppsala, Sweden

${ }^{\dagger}$ Students of the 'Evolution and Development' Course 2013 (course code: 1BG397) in alphabetic order; these authors contributed equally to the work.

e-mail addresses:

JEL:

Jordi.Estefa_Lopez.1555@student.uu.se;

SEL:

Simon.Liedholm_Eckerstrom.5353@student.uu.se; AAL: antonialochte@aol.com; MBO: marta_oliveira1990@hotmail.com; MP: paziomagdalena@gmail.com; JPM: jesuspm92@gmail.com; PRM: Patrik.Rodin_Morch.4091@student.uu.se; SS: sssala@utu.fi; JY: juliamyork@gmail.com; AY: andrewyo@hawaii.edu

*Corresponding author

Ralf Janssen

Phone: $\quad(++46) 184712763$

e-mail: $\quad$ ralf.janssen@geo.uu.se

Word count: 6494 (including supplementary data)

Figures: $\quad 7$

Tables:

Supplementary data: 1 additional figure, additional text

Keywords: arthropod limb development, evolution, limb patterning 


\begin{abstract}
A current hypothesis states that the ancestral limb of arthropods is composed of only two segments. The proximal segment represents the main part of the modern leg, and the distal segment represents the tarsus and claw of the modern leg. If the distal part of the limb is an ancestral feature, one would expect conserved regulatory gene networks acting in distal limb development in all arthropods and possibly even their sister-group, the onychophorans. We investigated the expression patterns of six genes known to function during insect distal limb development in the onychophoran Euperipatoides kanangrensis, i.e., clawless (cll), aristaless (al), spineless (ss), zinc finger homeodomain 2 (zfh2), rotund ( $r n$ ) and Lim1. We find that all investigated genes are expressed in at least some of the onychophoran limbs. The expression patterns of most of these genes, however, display crucial differences to the known insect patterns. The results of this study question the hypothesis of conserved distal limb evolution in arthropods and highlight the need for further studies on arthropod limb development.
\end{abstract}




\section{Introduction}

The arthropods make up one of the most diverse group of organisms on the planet. The triumphant march of the arthropods began as early as the lower Cambrian some 520 Mya (if not earlier) (e.g. Budd and Jensen 2000; Edgecombe 2010). At some point before their massive radiation, the arthropods evolved jointed limbs (hence the name Arthropoda) from a likely lobopod-like ancestral limb (reviewed in Shubin et al. 1997). This evolutionary novelty was probably one of the main reasons arthropod species became as successful as they are today, in both number and diversity. Segmentation (podomerization) of the limbs was a prerequisite for a much more advanced and functional way of locomotion, and also may have allowed the developmental modification of single podomeres without necessarily affecting neighboring podomeres. This enabled the evolution of countless modified limb-types in arthropods. The segmented limbs of arthropods most likely evolved from a much simpler tube-like lobopod appendage as represented by the appendages of recent onychophorans and tardigrades. These two phyla represent the closest living relatives to the Arthropoda, and recent studies support the idea that the onychophorans, rather than the tardigrades, are the most closely related arthropod out-group (Dunn et al. 2008; Budd and Telford 2009; Campbell et al. 2011; Giribet and Edgecombe 2012).

Data on onychophoran limb development will allow us to decipher, or at least speculate on, the ancestral mode of limb development in arthropods, as such features are most likely conserved in onychophorans, if at all. A previous study investigating the expression of the so-called limb gap genes (Rauskolb 2001) in E. kanangrensis found that their expression is highly conserved in arthropods and onychophorans. This data suggests functional homology of the limbs as walking appendages and, somewhat surprisingly, that limb patterning predates limb-segmentation (Janssen et 
al. 2010). Determination of the anterior-posterior axis of the limbs seems, as in Drosophila melanogaster (Cohen 1990), to be achieved through the highly conserved segment polarity gene network, including the function of genes such as engrailed (en), hedgehog (hh), and wingless (wg) (Eriksson et al. 2009; Janssen and Budd 2013). To investigate onychophoran limb development and the origin of limb patterning further, we cloned the orthologs of six distal-limb patterning genes from $E$. kanangrensis and documented their expression patterns during limb development. These genes are clawless (cll), aristaless (al), rotund ( $r n$ ), zinc finger homeodomain 2 (zfh2), spineless (ss), and Lim1. They are all directly or indirectly involved in the formation of the distal region in Drosophila limbs (Lecuit and Cohen 1997; Dear and Rabbitts 1994; Kojima et al. 2005; Pueyo et al. 2000; Galindo et al. 2002; Duncan et al. 1998; Guarner et al. (in press)). In Drosophila, the proximodistal (PD) axis of the walking limbs is regionalized by the action of leg gap genes such as dachshund (dac), Distal-less ( $\mathrm{Dll}$ ) and by FGF signaling (Galindo et al. 2002; reviewed in Rauskolb 2001 and Kojima 2004). The latter activates Liml, Bar, and al and represses the expression of $r n$ in posterior adjacent cells (Galindo et al. 2002). The sub-distal domain of $r n$ is proximally determined by the repressive function of dac. Lim1 then represses Bar via the activation of $a l$ and $c l l$, which act as direct repressors of Bar (Galindo et al. 2002; Kojima et al. 2005). As a result of this interaction, $c l l$, al, and Liml are expressed in the pretarsal region, and Bar and $r n$ are expressed in the adjacent tarsal region.

If the distal regions of arthropod and onychophoran limbs are homologous, we would expect these genes, or at least a number of them, to be expressed in comparable patterns in the distal limbs of the onychophoran. We find, however, that their 
expression patterns in the limbs diverge from that in insects, although all of these genes are expressed in at least a subset of the onychophoran appendages.

\section{Methods}

Embryo collection, fixation and staging

Embryos were collected and fixed as described in Janssen et al. (2010). Embryos were staged according to Janssen and Budd (2013).

\section{Gene cloning}

Total RNA was isolated from E. kanangrensis embryos of different stages using TRIZOL (Invitrogen). In a subsequent step poly-A RNA was extracted from total RNA (PolyATtract mRNA isolation system III, Promega) and reverse transcribed into cDNA (SuperscriptII first strand synthesis system for RT-PCR, Invitrogen). Fragments of onychophoran clawless (cll), aristaless (al), spineless (ss), zinc finger homeodomain 2 (zfh2), rotund ( $r n)$, and Lim1 were isolated by means of PCR with gene specific primers (based on a sequenced embryonic transcriptome (Janssen and Budd 2013); a list of the primer sequences is provided in the supplementary material). In all cases a first PCR was followed by a second (nested) PCR. Sequences of all fragments were determined from both strands on a 3100 automated sequencer, Terminator Cycle Sequencing Kit; Perkin-Elmer Applied Biosystems, Foster City, CA, USA) using Big Dye dye-terminators version 3.1 (Big Dye Terminator Cycle Sequencing Kit; Perkin-Elmer Applied Biosystems, Foster City, CA, USA), and cloned into pCR-II vectors (TA Cloning Kit Dual Promoter, Invitrogen, Carlsbad, Ca, USA). Isolated sequences are available under accession numbers HG794495 (Ek-cll), HG794496 (Ek-al), HG794500 (Ek-ss), HG794499 (Ek-rn), HG794498 (Ek-zfh2), and 
HG794497 (Ek-Lim1).

Whole mount in situ hybridization and nuclear staining

In situ hybridization was performed as described by Tautz and Pfeifle (1989). Digoxigenin-labeled RNA probes were transcribed from the cloned fragments. E. kanangrensis embryos were hybridized with the probes at $65^{\circ} \mathrm{C}$ for at least 16 hours. No protein-K treatment and no additional fixation were performed. Washing and incubation steps were generally prolonged because of the large size of the embryos. A detailed step-by-step protocol is provided as supplementary text. Nucleic staining was done by incubation of the embryos in $1 \mathrm{~g} / \mathrm{ml}$ of the fluorescent dye 4-6-diamidin-2phenylindol (DAPI) in phosphate-buffered saline with $0.1 \%$ Tween-20 (PBST) for 20 -30 minutes.

\section{Data documentation}

Embryos were analyzed under a Leica dissection microscope equipped with a Leica DC100 digital camera. The image processing software Adobe Photoshop CS2 (Version 9.0.1 for Apple Macintosh) was used for linear corrections of brightness, contrast, and color values in all images.

\section{Results}

Sequence analysis

We aligned, by hand, the homeodomain(s) of Euperipatoides clawless (cll), aristaless (al), Lim1, and zinc finger homeodomain 2 (zfh2), the zinc finger of rotund ( $r n)$, and the PAS domains of spineless (ss) with the corresponding sequences from the 
myriapod Glomeris marginata (best BLAST hit found in an embryonic transcriptome (Janssen and Budd 2013)) and the published sequences from Drosophila melanogaster, Tribolium castaneum, and other arthropods (supplementary figure Fig. S1). In order to show orthology of the Euperipatoides genes, we compared these sequences with the sequences of the most-related genes from Drosophila and Tribolium (supplementary figure Fig. S1). It shows that the onychophoran sequences are more similar to their putative orthologs than to the related genes with the second best hit found via BLAST search in the genomes of Drosophila and Tribolium.

\section{Expression patterns}

clawless $(\mathrm{cll})$ is first expressed at around stage 14 when it appears in the ectoderm of the tips of the slime papillae, the most anterior walking limbs, and very faintly in the jaws (Fig. 1A). Expression in the limbs thus appears clearly after the beginning of limb outgrowth which starts at approximately stage 12 (Janssen and Budd 2013). At subsequent stages, expression in the jaw becomes stronger and additional walking limbs begin to express $c l l$ in their tips (Fig. 1B/C). Initially, $c l l$ expression is restricted to the tips of the limbs, but later during development it is also inside the limbs (Fig. $1 \mathrm{D} / \mathrm{E})$. The expression pattern in the slime papillae is similar to that in the walking limbs. Expression in the jaw, however, is located posteriorly in the ectoderm (Fig. 1BD, and summarized in Fig. 7) and hence the very tips of these appendages do not express cll. The frontal appendages never express $c l l$ at the investigated developmental stages (i.e. all stages as described in Janssen and Budd (2013)). Apart from the expression in the limbs, $c l l$ is also expressed in the developing dorsal tube (= heart) (Fig. 1B) and temporarily between the bases of the limbs (Fig. 1B); this latter 
expression is weak between jaw and slime papilla, and between the slime papilla and the first walking limb.

aristaless (al), like $c l l$, is first expressed around stage 14, when it appears faintly in all limbs except the frontal appendages (Fig. 2A). This first expression is clearly in the ectoderm of the tips of the limbs (Fig. 2B). Later, expression appears in the frontal appendages and slime papillae, where it is expressed in a leg gap-gene-like domain in the center of these limbs while expression remains in the tips of the jaws and the walking limbs (Fig. 2C-F and 7). At these later stages, expression in the tips of the walking limbs is relatively faint compared to expression in the other appendages (Fig. 2D-F).

Expression of $\operatorname{Liml}$ is first detectable in the primordium of the complete jaw-bearing segment (Fig. 3A-G). While the frontal appendages do not express Liml at early stages, it is expressed in the ectoderm of the complete jaws, except the most distal tips, and in the mesoderm of the tips of the slime papillae and the walking limbs (Fig. 3B-D). A single dot of expression is located ventrally to the base of the slime papillae and the walking limbs (Fig. 3D). The described expression persists throughout development (Fig. 3E). At approximately stage 20, expression appears inside (mesoderm) of the frontal appendages (Fig. 3F-H), and in the ectoderm of the tips of the slime papillae and the walking limbs (Fig. 3I, J). The mesodermal expression is likely correlated to the formation of the nephridia, as indicated by the larger domain of Lim1-expressing tissue within the walking limbs of L4 and L5, in which the nephridia are enlarged (Mayer 2006).

rotund $(\mathrm{rn})$ is expressed in the tips of the frontal appendages from approximately stage 14 onwards (Fig. 4A, B). At the same time, strong expression appears in the anterior of the head lobes (Fig. 4A, B). There is very faint expression in the most 
posterior region of the embryo (Fig. 4A). Somewhat later, expression appears in the tips of the jaws and in the complete frontal appendages, where expression in the tips is stronger (Fig. 4D). Weak expression appears in the tips of the slime papillae and the walking limbs (Fig. 4C-F). Patches of expression are located posterior at the base of jaws, slime papillae, and walking limbs (Fig. 4C, E-F). At very late stages, a complex pattern of $r n$ appears in the ventral nervous system and in numerous dots in the tissue dorsal to the limbs (Fig. 4G); this later expression may be correlated with the formation of body-papillae (cf. Walker and Tait 2004).

zinc finger homeodomain 2 ( $z f h 2)$ is first expressed in the complete head lobes (Fig. 5A, B) and the developing ventral nervous system (Fig. 5A). Later, expression is ubiquitous in the frontal appendages and the other limbs. The area around the base of the frontal appendages and ventral at the bases of the other limbs shows no or very weak expression (Fig. 5C). At approximately stage 17, a sub-distal ring of stronger expression appears in the otherwise ubiquitously $z f h 2$-expressing slime papillae and walking limbs (Fig. 5D-F). Such rings are absent from the frontal appendages and jaws. The jaws do not (or only faintly) express $z$ fh 2 in their tips, while the proximal part of the jaws expresses $z h f 2$ strongly (Fig. 5E). The area around the base of the frontal appendages remains free from expression.

spineless (ss) is first detectable at stage 13 in the tips of the frontal appendages (Fig. 6A). Later, expression appears in the tips of all appendages (Fig. 6B-F). In the slime papillae, this expression is not at the very tip but somewhat towards the posterior; in the jaw, expression is likewise shifted, but towards the anterior (Fig. 6E). Expression is now in the complete frontal appendages (except the most proximal tissue), but expression in their tips is still enhanced (Fig. 6B, C, c). Thin rings of stronger expression corresponding with the grooves of the annuli are visible (Fig. 6C,c). Two 
dots of expression appear posterior to the bases of the frontal appendages in a position where the frontal processes will form (Walker and Tait 2004) (Fig. 6C). At later stages, the complete body of the embryo is covered with numerous spots of ssexpression that is inter alia associated with the forming frontal processes and the oral lips (Fig. 6G). At later developmental stages, four dots of expression are located in the ectoderm of the slime papillae and the walking limbs (Fig. 6H, I). Thin ss-positive lines run at the interface between mesodermal and ectodermal tissue that connect these dots. It is likely that this expression is basally in ectodermal cells. Such expression was also reported for some of the limb gap genes (Janssen et al. 2010). It may either represent a feature of gene expression in the limbs, or a staining/fixation artifact.

\section{Discussion}

Expression in insects and onychophorans - a mixture of conserved and diverged patterns

The investigated limb-patterning genes have almost exclusively been studied in the model insects Drosophila melanogaster and Tribolium castaneum. Data from noninsect species is restricted to the expression pattern analysis of $s s$ in the myriapod Glomeris marginata (Janssen 2013). Data from crustaceans and chelicerates are completely lacking.

In Drosophila, the aristaless (al) gene is responsible for the patterning of the distalmost limb-structures, i.e. the arista of the antenna and the pretarsal claw of the walking limb (Lecuit and Cohen 1997). This function is conserved in the beetle Tribolium castaneum (Beermann and Schröder 2004), and so is the expression in the cricket Gryllus bimaculatus (Miyawaki et al. 2002). 
Expression of Euperipatoides al is possibly conserved in the jaws and the walking limbs where it is expressed in the tips as in insects. In the frontal appendages and the slime papillae, however, the detected central expression domain is reminiscent of that of a typical limb gap gene (cf. expression of e.g. dachshund (dac) (Janssen et al. 2010)), and therefore is clearly different from the expected expression in the tips. Compared to $d a c$, al is expressed in a broader domain in the slime papillae; Euperipatoides dac is not expressed in the frontal appendages. It may thus be that the function of $a l$ in the frontal appendages and slime papillae is that of a leg gap gene supplementing (or replacing) the function of the known arthropod leg gap genes.

clawless (cll) (aka C15 (Dear and Rabbitts 1994)) is expressed in the pretarsal (claw) region of the Drosophila walking limb-disc and the arista of the eye-antennal disc (Campbell 2005; Kojima et al. 2005). cll is co-expressed with al, and it has been shown that these two genes cooperatively interact to repress a pair of Bar genes (BarH1 and BarH2 (Higashijima et al. 1992)) in the adjacent podomere, the distal tarsus (Campbell 2005; Kojima et al. 2005). In Tribolium, cll is expressed in a conserved pattern in the tips of all appendages except the mandibles (Grossmann and Prpic 2009; Prpic pers. com.); RNAi knock-down studies of cll during metamorphosis did not result in any recognizable phenotype in the antennae of adult Tribolium (Angelini et al. 2009).

Among all investigated genes, the expression profile of Euperipatoides cll is most similar to that in insects, as it is expressed in the tips of all appendages (except the frontal appendages). It is therefore likely that its function as a distal limb gene is conserved in onychophorans and arthropods.

The Liml gene is also expressed in the pretarsal podomere of the Drosophila walking legs and the arista of the antennae (Pueyo et al. 2000; Tsuji et al. 2000). Apart from 
that, it is also expressed in three domains along the proximodistal (PD) axis. In the tip of the limbs, it is likely activated by al and cll (Campbell 2005; Kojima et al. 2005). In Tribolium, Lim 1 is not involved in the formation of the distal tip of the antenna or the other limbs as shown by RNAi mediated knock-down experiments (Angelini et al. 2009, 2012). Embryonic expression patterns and function of Tribolium Liml have, however, not been studied yet.

The different function of Lim 1 in distal limb patterning in Drosophila and Tribolium already indicates that the function of this gene may be highly flexible. Data from insects suggest that either the distal-tip function of Lim 1 has been lost in Tribolium or has been gained within the lineage leading to Drosophila. The expression of Euperipatoides Liml in the limbs is complex and only partly comparable to that in Drosophila. In fact, only the late ectodermal expression in the tips of the slime papillae and the walking limbs is similar to that in Drosophila. The lack of Lim1 in the tips of the jaws and the exclusively (and late) mesodermal expression in the frontal appendages are not comparable to the expression data from Drosophila.

In Drosophila, the $r n$ gene is transiently expressed in a ring corresponding to the tarsal region of the developing limb and in the mutant phenotype tarsal segments are missing (Galindo et al. 2002). Compared to $\mathrm{al}$, $\mathrm{cll}$, and Liml, $r n$ is thus expressed in a more proximal region of the developing limb. Knock-down of $r n$ in Tribolium frequently leads to disturbed development of the funicle and the clubs of the antennae (Angelini et al. 2009). Although the function of $r n$ influences comparable regions of the antennae in Tribolium and Drosophila, its function appears to be different and affects areas of different size (Angelini et al. 2009). In the walking limbs of Tribolium, knock-down of $r n$ leads to reduced tarsi and the loss of the tarsal joints 
(Angelini et al. 2012), a phenotype that is comparable to that in Drosophila. The mRNA expression of Tribolium $r n$ is unknown.

The expression of $r n$ in the onychophoran indicates a function in distal limb development. This, however, would be different from its function in insects, since in insects the expression is proximally adjacent and not overlapping with the expression of $a l, c l l$, and Liml. The regulatory interaction of these genes and the tissue they pattern are therefore different in insects and onychophorans.

In Drosophila, the expression of zfh 2 is largely restricted to the developing nervous system (Lai et al. 1991). It plays, however, a role during distal limb patterning as well, where it controls Notch signaling and apoptosis in tarsal segments (Guarner et al. (in press)). In Tribolium, zfh2 is transiently expressed in the tips of all limbs and in RNAi-mediated knock-down phenotypes the claws are often malformed (Kittelmann et al. 2009). In addition to that, the joint between the femur and the tibiotarsus is not formed in RNAi-treated embryos (Kittelmann et al. 2009). The overall expression pattern of onychophoran $z f h 2$ resembles very much that of Tribolium $z f h 2$, in that it is first expressed in the head lobes and then in the developing ventral nervous system, without expression in the posterior segment addition zone and newly added posterior segments. In the limbs, however, the expression is significantly different. While $z$ fh 2 is expressed in the tips of the limbs in Tribolium, this expression is weak or lacking in Euperipatoides, indicating that the tip-forming function of Tribolium zfh2 is likely not present in the onychophoran.

In Drosophila, loss of function studies of spineless ( $s s$ ) have shown that most of the tarsal podomeres fail to form, that the antennae transform into walking limb-like structures, and that most bristles fail to develop properly (Duncan et al. 1998; Emmons et al. 1999). In Tribolium, ss is strongly expressed in the antennae and more 
weakly in the palps of the maxillae and the labium (Toegel et al. 2009; Shippy et al. 2009). Later, it is also expressed as a ring in the thoracic limbs roughly corresponding with the junction of the coxa and the trochanter (Toegel et al. 2009). Comparable expression in the tips has also been reported for the antennae and the maxillae of the myriapod Glomeris marginata (Janssen 2013). It seems therefore likely that the function in limb patterning is at least partially conserved in mandibulate arthropods. In Euperipatoides, ss is strongly expressed in the developing frontal appendages, which are the functional equivalent to the insect antennae, although it is not the appendage of the homologous segment (Eriksson et al. 2003; Eriksson et al. 2010). It appears thus that enhanced expression of $s s$ is pesent in appendages with antennal morphology and sensory function in lobopods and arthropods. The temporal expression in the tips of all appendages also indicates that the function in tip development may be conserved in onychophorans and arthropods. The late expression of $s s$ is clearly associated with the formation of bristles, and thus all major aspects of ss-function in Drosophila are likely conserved in the onychophoran.

\section{Different appendages - different expression patterns}

We find that the expression patterns of the investigated distal limb patterning genes differ considerably in the different types of onychophoran appendages. Most conservation compared to the expression patterns in insects is seen in the walking limbs (summarized in Fig. 7). This accords with the idea that the walking limb represents the ancestral form compared to the modified head appendages (Struhl 1982; Duncan et al. 1998). The clearest aberration from the patterning seen in the distal walking limb is found in the frontal appendages (cf. also expression of limb gap genes (Janssen et al. 2010)). These differences are, however, not much more 
pronounced than those found in the jaws and the slime papillae; and a recent study revealed likely homology of the walking limb claws with the jaw blades in onychophorans (Oliveira and Mayer 2013), which would suggest similar genetic patterning of these distal structures. This finding is of potential importance, because similar derived gene expression patterns also occur in the arthropod labrum, a structure of still disputed origin and homology. The argument that partially different expression patterns in limbs such as the arthropod labrum and the onychophoran frontal appendage would imply non-homology to the other limbs is thus not valid. That is homology of the frontal appendages to the other onychophoran limbs was never sincerely questioned, different from the situation of the arthropod labrum (e.g. Budd 2002, Scholtz and Edgecombe 2006, Kimm and Prpic 2006, Posnien et al. 2009).

How 'ancestral' is the ancestral distal limb patterning system?

The work of Casares and Mann (2001) strongly implies that the distal region of the arthropod limb represents part of an ancestral ground state of limb morphology. They show that the loss of limb-identity selector genes transforms the walking limbs of Drosophila into two-segmented limbs composed of a proximal region representing all podomeres except the tarsus and a distal tarsal segment (Casares and Mann 2001). Our working hypothesis was therefore that if this idea holds true, one would expect largely conserved expression patterns in other arthropod groups, and possibly even in the arthropod sister-group, the onychophorans. Our results, however, are ambiguous. Despite our findings of potentially conserved patterns of $c l l, L i m 1, a l$, and ss (mostly in the tips of the walking limbs), we also find divergent patterns of $z f h 2$ and $r n$. This means that either the investigated genes are part of an ancestral patterning network 
that has been altered in the lineage leading to insects, or that the onychophoran patterns are derived, or that distal limb patterning has evolved independently in onychophorans and arthropods.

\section{Conclusions}

The expression patterns of the reported limb-patterning genes display a number of conserved as well as derived features. The currently available data therefore are not conclusive on whether the distal limb-patterning network is principally conserved in arthropods and onychophorans. The analysis of the onychophoran data is hampered by the lack of comparative data from non-insect arthropods and basally branching insects. Therefore more data on the expression, and if possible the function, of these genes are needed to "follow" evolution of the distal limb patterning system along the phylogenetic tree. We suggest investigation of the same genes in at least one basally branching insect, one crustacean, one myriapod, and one chelicerate. Such data will likely allow more conclusive inferences on the predicted evolutionary robustness of the distal limb patterning network in arthropods and their closest relatives, the onychophorans.

\section{Acknowledgements}

The experiments were conducted during the "Evolution and Development" course at Uppsala University in spring 2013. We gratefully acknowledge the support of the NSW Government Department of Environment and Climate Change by provision of a permit SL100159 to collect onychophorans at Kanangra Boyd National Park and to the Australian Government Department of the Environment, Water, Heritage, and the Arts for export permits WT2009-4598 and WT2012-4704. The authors wish to thank 
Jean Joss, Rolf Ericsson, Robyn Stutchbury, and especially Noel Tait, for their help during onychophoran collection. Mattias Hogvall is thanked for his help as laboratory assistant. Nico Posnien and Alistair McGregor helped with the analysis of the embryonic transcriptome.

\section{References}

Angelini DR, Kikuchi M, Jockusch EL (2009) Genetic patterning in the adult capitate antenna of the beetle Tribolium castaneum. Dev Biol 327:240-251

Angelini DR, Smith FW, Jockusch EL (2012) Extent With Modification: Leg patterning in the beetle Tribolium castaneum and the evolution of serial homologs. G3 (Bethesda) 2:235-248

Beermann A, Schroeder R (2004) Functional stability of the aristaless gene in appendage tip formation during evolution. Dev Genes Evol 214:303-308

Budd GE, Jensen S (2000) A critical reappraisal of the fossil record of the bilaterian phyla. Biol Rev Camb Philos Soc 75:253-295

Budd GE (2002) A palaeontological solution to the arthropod head problem. Nature 417:271-275

Budd GE, Telford MJ (2009) The origin and evolution of arthropods. Nature 457:812817

Campbell G, Tomlinson A (1998) The roles of the homeobox genes aristaless and Distal-less in patterning the legs and wings of Drosophila. Development $125: 4483-4493$

Campbell G (2005) Regulation of gene expression in the distal region of the Drosophila leg by the Hox11 homolog, C15. Dev Biol 2005 278:607-618 
Campbell LI, Rota-Stabelli O, Edgecombe GD, Marchioro T, Longhorn SJ, Telford MJ, Philippe H, Rebecchi L, Peterson KJ, Pisani D (2011) MicroRNAs and phylogenomics resolve the relationships of Tardigrada and suggest that velvet worms are the sister group of Arthropoda. Proc Natl Acad Sci USA $108: 15920-15924$

Casares F, Mann RS (2001) The ground state of the ventral appendage in Drosophila. Science 293:1477-1480

Cohen SM (1990) Specification of limb development in the Drosophila embryo by positional cues from segmentation genes. Nature 343:173-177

Dear TN, Rabbitts TH (1994) A Drosophila melanogaster homologue of the T-cell oncogene HOX11 localises to a cluster of homeobox genes. Gene 141:225229

Duncan DM, Burgess EA, Duncan I (1998) Control of distal antennal identity and tarsal development in Drosophila by spineless-aristapedia, a homolog of the mammalian dioxin receptor. Genes Dev 12:1290-1303

Dunn CW, Hejnol A, Matus DQ, Pang K, Browne WE, Smith SA, Seaver E, Rouse GW, Obst M, Edgecombe GD, Sorensen MV, Haddock SHD, Schmidt-Rhaesa A, Okusu A, Kristensen RM, Wheeler WC, Martindale MQ, Giribet G (2008) Broad phylogenomic sampling improves resolution of the animal tree of life. Nature 452:745-749

Edgecombe GD (2010) Arthropod phylogeny: an overview from the perspectives of morphology, molecular data and the fossil record. Arthropod Struct Dev $39: 74-87$

Emmons RB, Duncan D, Estes PA, Kiefel P, Mosher JT, Sonnenfeld M, Ward MP, Duncan I, Crews ST (1999) The Spineless-Aristapedia and Tango bHLH-PAS 
proteins interact to control antennal and tarsal development in Drosophila. Development 126:3937-3945

Eriksson BJ, Tait NN, Budd GE (2003) Head Development in the Onychophoran Euperipatoides kanangrensis With Particular Reference to the Central Nervous System. J Morphol 255:1-23

Eriksson BJ, Tait NN, Budd GE, Akam M (2009) The involvement of engrailed and wingless during segmentation in the onychophoran Euperipatoides kanangrensis (Peripatopsidae: Onychophora) (Reid 1996). Dev Genes Evol 219:249-264

Eriksson BJ, Tait NN, Budd GE, Janssen R, Akam M (2010) Head patterning and Hox gene expression in an onychophoran and its implications for the arthropod head problem. Dev Genes Evol 220:117-122

Galindo MI, Bishop SA, Greig S, Couso JP (2002) Leg patterning driven by proximal-distal interactions and EGFR signaling. Science. 297:256-259

Giribet G, Edgecombe GD (2012) Reevaluating the arthropod tree of life. Annu Rev Entomol 57:167-186

Grossmann D, Prpic NM (2012) Egfr signaling regulates distal as well as medial fate in the embryonic leg of Tribolium castaneum. Dev Biol 370 264-272

Guarner A, Manjon C, Edwards K, Steller H, Suzanne M, Sanchez-Herrero E (in press) The zinc finger homeodomain-2 gene of Drosophila controls Notch targets and regulates apoptosis in the tarsal segments. Dev Biol. doi:pii: S0012-1606(13)00555-1. 10.1016/j.ydbio.2013.10.011.

Higashijima S, Kojima T, Michiue T, Ishimaru S, Emori Y, Saigo K (1992) Dual Bar homeo box genes of Drosophila required in two photoreceptor cells, R1 and R6, and primary pigment cells for normal eye development. Genes Dev 6:50- 
Janssen R, Eriksson BJ, Budd GE, Akam M, Prpic NM (2010) Gene expression patterns in an onychophoran reveal that regionalization predates limb segmentation in pan-arthropods. Evol Dev 12:363-372

Janssen R (2013) Developmental abnormalities in Glomeris marginata (Villers 1789) (Myriapoda: Diplopoda): implications for body axis determination in a myriapod. Naturwissenschaften 100:33-43

Janssen R, Budd GE (2013) Deciphering the onychophoran 'segmentation gene cascade': gene expression reveals limited involvement of pair rule gene orthologs in segmentation, but a highly conserved segment polarity gene network. Dev Biol 382:224-234

Kimm MA, Prpic NM (2006) Formation of the arthropod labrum by fusion of paired and rotated limb-bud-like primordia. Zoomorphology 125:147-155

Kittelmann M, Schinko JB, Winkler M, Bucher G, Wimmer EA, Prpic NM (2009) Insertional mutagenesis screening identifies the zinc finger homeodomain 2 (zfh2) gene as a novel factor required for embryonic leg development in Tribolium castaneum. Dev Genes Evol 219:399-407

Kojima T (2004) The mechanism of Drosophila leg development along the proximodistal axis. Dev Growth Differ 46:115-129

Kojima T, Tsuji T, Saigo K (2005) A concerted action of a paired-type homeobox gene, aristaless, and a homolog of Hox11/tlx homeobox gene, clawless, is essential for the distal tip development of the Drosophila leg. Dev Biol 279:434-445

Lai Z, Fortini ME, Rubin GM (1991) The embryonic expression patterns of $z f h-1$ and zfh-2, two Drosophila genes encoding novel zinc-finger homeodomain 
proteins. Mech Dev 34:123-134

Lecuit T, Cohen SM (1997) Proximal-distal axis formation in the Drosophila leg. Nature 388:139-145

Mayer G (2006) Origin and differentiation of nephridia in the Onychophora provide no support for the Articulata. Zoomorphology 125:1-12

Miyawaki K, Inoue Y, Mito T, Fujimoto T, Matsushima K, Shinmyo Y, Ohuchi H, Noji S (2002) Expression patterns of aristaless in developing appendages of Gryllus bimaculatus (cricket). Mech Dev 113:181-184

Oliveira IS, Mayer G (2013) Apodemes associated with limbs support serial homology of claws and jaws in Onychophora (velvet worms). J Morph $274: 1180-1190$

Posnien N, Bashasab F, Bucher G (2009) The insect upper lip (labrum) is a nonsegmental appendage-like structure. Evol Dev 11:480-488

Pueyo JI, Galindo MI, Bishop SA, Couso JP (2000) Proximal-distal leg development in Drosophila requires the apterous gene and the Lim1 homologue dlim1. Development 127(:5391-5402

Rauskolb C (2001) The establishment of segmentation in the Drosophila leg. Development 128:4511-4521

Scholtz G, Edgecombe GD (2006) The evolution of arthropod heads: reconciling morphological, developmental and palaeontological evidence. Dev Genes Evol 216:395-415

Shippy TD, Yeager SJ, Denell RE (2009) The Tribolium spineless ortholog specifies both larval and adult antennal identity. Dev Genes Evol 219:45-51

Shubin N, Tabin C, Carroll S (1997) Fossils, genes and the evolution of animal limbs. Nature 388:639-648 
Struhl G (1982) Genes controlling segmental specification in the Drosophila thorax. Proc Natl Acad Sci 79:7380-7384

Tautz D, Pfeifle C (1989) A non-radioactive in situ hybridization method for the localization of specific RNAs in Drosophila embryos reveals translational control of the segmentation gene hunchback. Chromosoma 98:81-85

Toegel JP, Wimmer EA, Prpic NM (2009) Loss of spineless function transforms the Tribolium antenna into a thoracic leg with pretarsal, tibiotarsal, and femoral identity. Dev Genes Evol 219:53-58

Tsuji T, Sato A, Hiratani I, Taira M, Saigo K, Kojima T (2000) Requirements of Lim1, a Drosophila LIM-homeobox gene, for normal leg and antennal development. Development 127:4315-4323

Walker MH, Tait NN (2004) Studies of embryonic development and the reproductive cycle in ovoviviparous Australian Onychophora (Peripatopsidae). J Zool Lond $264: 333-354$

\section{Figure legends}

Fig. 1 Expression of Euperipatoides clawless ( $\mathrm{cll})$

In all panels anterior is to the left. A Lateral view. Beginning expression in the limbs. B Lateral view. Expression in limbs and heart. C Ventral view of same embryo shown in B. D Close-up on jaw and slime papilla. E Close-up on a walking limb. Abbreviations: fap, frontal appendage; hl, head lobe; j, jaw; h, heart; L, walking limb; sp, slime papillae

Fig. 2 Expression of Euperipatoides aristaless (al) 
A Ventro-dorsal view. Anterior is to the left. Beginning expression in the appendages. B Close-up on walking limbs of the same embryo shown in A. C Expression in the frontal appendage. Ventral view. Anterior is up. D Expression in the jaw. Ventral view. Anterior is up. E Expression in the slime papilla. Ventral view. Anterior is up. F Expression in the walking limb. Ventral view. Anterior is up. $\mathbf{A}^{\prime}$ shows DAPI staining of the same embryo shown in A. Abbreviations as in Fig. 1.

Fig. 3 Expression of Euperipatoides Lim1

In all panels anterior is to the left. A Ventral view. Expression in the jaw-bearing segment. B Ventral view. C Ventral view. Close-up of anterior region of embryo shown in B. Note that the tip of the jaw is free from expression. Expression in the slime papilla and the walking limb is mesodermal (arrowheads). D Lateral view of same embryo shown in B. Note dot-like expression ventral to the base of the limbs (arrows). E Lateral view. Arrow as in D. F Lateral view. G Ventral view of same embryo as shown in F. H Mesodermal expression in the frontal appendage. I Close-up on the jaw and the slime papilla. J Close-up on the first two walking limbs. $\mathbf{A}^{\prime}$ shows DAPI staining of the same embryo shown in A. Abbreviations as in Fig. 1.

Fig. 4 Expression of Euperipatoides rotund ( $r n)$

In all panels anterior is to the left. A Ventral view. Beginning expression in the frontal appendages and a domain anterior in the head lobes. B Lateral view. Same embryo as in A. C Ventral view on anterior part of an embryo. Expression in the tips of the jaw, the slime papilla, and weakly in the walking limbs. D-F Close-ups on frontal appendage (D), walking limbs (E), and jaw and slime papilla (F). G Lateral view. Note complex pattern of expression in tissue dorsal (arrowheads) and ventral 
(asterisks) to the limbs. $\mathbf{A}^{\prime}$ shows DAPI staining of the same embryo as in A. Abbreviations as in Fig. 1, and saz, segment addition zone. Abbreviations as in Fig. 1.

Fig. 5 Expression of Euperipatoides zinc finger homeodomain 2 (zfh2)

In all panels anterior is to the left. A Lateral view. Arrowhead points to lack of expression between head lobe and jaw-bearing segment. Arrow points to expression in the ventral nervous system. Note that staining in the extraembryonic tissue of the embryo is likely artificial (asterisks). B Ventral view. Arrowhead as in A. C Ventral view. Arrow as in A. D Dorsal view. E Ventral view. Close up on head lobe and jaw. F Ventral view. Close-up on slime papilla and walking limbs. Same embryo as in D. $\mathbf{A}^{\prime}$ shows DAPI staining of the same embryo as in A. Abbreviations as in Fig. 1.

Fig. 6 Expression of Euperipatoides spineless (ss)

In all panels anterior is to the left. A Ventral view. Beginning expression in the tips of the frontal appendages. B Lateral view. Expression appears in the tips of all appendages. C Ventral view. Same embryo as in B. Arrowheads point to dot-like expression in the frontal processes. c Lateral view on anterior of another embryo (same stage). Note that the frontal appendage is bending backward. D-F Close-ups on walking limbs (D), jaw (E) and slime papilla (F). G Dorsal view. Arrowheads as in C. Arrows point to dots covering the complete body. g Ventral view. Close-up on the oral lips. Same embryo as in G. $\mathbf{H}$ and I Close ups on slime papilla (H) and walking limb (I). Same embryo as in G. $\mathbf{A}^{\prime}$ shows DAPI staining of the same embryo as in A. Abbreviations as in Fig. 1, and 1, oral lip.

Fig. 7 Schematic summary of expression patterns in the limbs 
Expression in the ectoderm is in RED, expression in the mesoderm is in GREEN (late expression) or in BLUE (early expression). DASHED RED line indicates ectodermal expression in a ring. LIGHT RED indicates weak expression in the ectoderm.

\section{Supplementary data}

Fig. S1 Alignments

Aligned are the conserved regions. For CLL, AL and LIM1 this is the HOMEODOMAINS; for RN this is the ZINC FINGER DOMAIN; for ZFH2 these are the three HOMEODOMAINS; for SS these are the two PAS domains. Dashes (-) indicate identical amino acids as in the onychophoran. Dots (.) indicate gaps in the alignment. Note the differences between the orthologs and other closely related genes. Abbreviations: Am, Apis mellifera (Insecta: Hymenoptera); Dm, Drosophila melanogaster (Insecta: Diptera); Dp, Daphnia pulex (Crustacea: Branchiopoda); Ek, Euperipatoides kanangrensis (Onychophora: Peripatopsidae); Gb, Gryllus bimaculatus (Insecta: Orthoptera); Gm, Glomeris marginata (Myriapoda: Diplopoda); Ma, Megaselia abdita (Insecta: Diptera); Nv, Nasonia vitripennis (Insecta: Hymenoptera); Tc, Tribolium castaneum (Insecta: Coleoptera). 